\title{
AVERAGING OF THE HAMILTON-JACOBI EQUATION IN INFINITE DIMENSIONS AND AN APPLICATION
}

\author{
SHIHONG WANG ${ }^{1}$ and ZUOYI ZHOU ${ }^{1}$
}

(Received 20 January 1997; revised 28 April 1998)

\begin{abstract}
We study the averaging of the Hamilton-Jacobi equation with fast variables in the viscosity solution sense in infinite dimensions. We prove that the viscosity solution of the original equation converges to the viscosity solution of the averaged equation and apply this result to the limit problem of the value function for an optimal control problem with fast variables.
\end{abstract}

\section{Introduction}

In this paper we are concerned with the averaging of the Hamilton-Jacobi-Bellman (HJB) equation with fast variables in $n$ sense in Hilbert space $X\left(X^{*}\right.$ is the dual of $X$ and $\left.X=X^{*}\right)$ :

$$
\begin{cases}V_{t}(t, x)+H\left(t, t / \varepsilon, x, V_{x}(t, x)\right)=0, & (t, x) \in[0, T) \times X \\ V(T, x)=g(x), & x \in X,\end{cases}
$$

where $\varepsilon \in R^{+} \equiv(0,+\infty) ; H$ and $g$ are given functions and satisfy conditions given in Section 2. For the definition of the viscosity solution of the HJB equation, refer to $[6,7]$. From [6,7], we know that equation (1.1) has a unique viscosity solution $V_{\varepsilon}(t, x)$, which satisfies

$$
\left|V_{\varepsilon}(t, x)-V_{\varepsilon}(\bar{t}, \bar{x})\right| \leq L_{\varepsilon}(|t-\bar{t}|+\|x-\bar{x}\|), \forall t, \bar{t} \in[0, T] ;\|x\|,\|\bar{x}\| \leq R
$$

with $R$ being a given constant and $L_{\varepsilon}$ a constant which is dependent on $\varepsilon$ and $R$ probably.

Our purpose in this paper is to study the limiting behavior of $V_{\varepsilon}(t, x)$ as $\varepsilon \rightarrow 0^{+}$. This problem has been studied by Chaplais [3] and Barron [1] in finite dimensions

${ }^{1}$ Institute of Mathematics, Fudan University, Shanghai, China

(C) Australian Mathematical Society 2000, Serial-fee code 0334-2700/00 
(that is, $X$ is $R^{n}$ ). The main difficulty in the passage from finite to infinite dimensions is to prove that the limit of $V_{\varepsilon}(t, x)$ exists. In $[1,3]$, the Arzela-Ascoli theorem has been used to deal with this, as a priori estimates of $V_{\varepsilon}(t, x)$ hold. However, in infinite dimensions, such a method does not apply again for there is no "appropriate" ArzelaAscoli theorem available. In this paper, we overcome this difficulty by using the properties of the viscosity solution itself and the perturbed test function method. We directly prove that if $L_{\varepsilon}$ is independent of $\varepsilon$, then $V_{\varepsilon}(t, x)$ converges to $V(t, x)$, which is a unique viscosity solution of the HJB equation

$$
\begin{cases}V_{t}(t, x)+\bar{H}\left(t, x, V_{x}(t, x)\right)=0, & (t, x) \in[0, T) \times X, \\ V(T, x)=g(x), & x \in X,\end{cases}
$$

where $\bar{H}:[0, T] \times X \times X^{*} \rightarrow R^{1}$ is defined by

$$
\bar{H}(t, x, p)=\int_{0}^{1} H(t, s, x, p) d s, \quad \forall(t, x, p) \in[0, T] \times X \times X^{*} .
$$

Next, we apply this result to study the limit problem of the value function of an optimal control problem with fast variables. The modeling of systems that have at least one component that oscillates rapidly is an important problem in optimal control theory. The motivation for this problem comes from the fact that the value function is used in the construction of feedback controls (see [9]).

\section{Averaging of the Hamilton-Jacobi equation}

First, let us make the following assumptions.

H1. $H:[0, T] \times R^{+} \times X \times X^{*} \rightarrow R^{1}$ and $g: X \rightarrow R^{1}$ are continuous; $H(t, \cdot, x, p)$ is periodic with period 1 . For any $t, \bar{t} \in[0, T] ; s, \bar{s} \in R^{+} ; x, \bar{x} \in X ; p, q \in X^{*}$, there exist constants $A_{1}, A_{2}, B_{1}, B_{2}, \bar{C}$ such that

$$
\begin{aligned}
& |H(t, s, x, p)-H(t, s, x, q)| \leq\left(A_{1}\|x\|+B_{1}\right)\|p-q\|, \\
& |H(t, s, x, p)-H(\bar{t}, \bar{s}, \bar{x}, p)| \\
& \quad \leq\left(A_{2}+B_{2}\|p\|\right)(|t-\bar{t}|+|s-\bar{s}|+\|x-\bar{x}\|), \\
& |g(x)-g(\bar{x})| \leq \bar{C}\|x-\bar{x}\| .
\end{aligned}
$$

H2. Let $\bar{H}:[0, T] \times X \times X^{*} \rightarrow R^{1}$ be a continuous function, which satisfies

$$
\bar{H}(t, x, p)=\int_{0}^{1} H(t, s, x, p) d s, \quad \forall(t, x, p) \in[0, T] \times X \times X^{*} .
$$


By assumption $\mathrm{H} 1$, for any $t, \bar{t} \in[0, T] ; x, \bar{x} \in X ; p, q \in X^{*}$, we have

$$
\begin{aligned}
& |\bar{H}(t, x, p)-\bar{H}(t, x, q)| \leq\left(A_{1}\|x\|+B_{1}\right)\|p-q\|, \\
& |\bar{H}(t, x, p)-\bar{H}(\bar{t}, \bar{x}, p)| \leq\left(A_{2}+B_{2}\|p\|\right)(|t-\bar{t}|+\|x-\bar{x}\|) .
\end{aligned}
$$

From $[6,7]$, we know equation (1.1) has a unique viscosity solution which satisfies (1.2), and equation (1.3) has a unique viscosity solution $V(t, x)$ satisfying

$$
|V(t, x)-V(\bar{t}, \bar{x})| \leq \bar{L}(|t-\bar{t}|+\|x-\bar{x}\|), \forall t, \bar{t} \in[0, T] ;\|x\|,\|\bar{x}\| \leq R
$$

with $R$ being a given constant and $\bar{L}$ a constant which is only dependent on $R$.

LEMMA 2.1. Let $\mathrm{H} 1, \mathrm{H} 2$ hold, $Z(t, x, y)=V_{\varepsilon}(t, x)-V(t, y), \forall(t, x, y) \in[0, T] \times$ $X \times X$; then $Z(\cdot, \cdot, \cdot)$ is a viscosity subsolution of the $\mathrm{HJB}$ equation

$$
\left\{\begin{array}{r}
Z_{t}(t, x, y)+H\left(t, t / \varepsilon, x, Z_{x}(t, x, y)\right)-\bar{H}\left(t, y,-Z_{y}(t, x, y)\right)=0, \\
\forall(t, x, y) \in[0, T) \times X \times X, \\
Z(T, x, y)=g(x)-g(y), \quad(x, y) \in X \times X .
\end{array}\right.
$$

The proof can be seen in [6] or [7].

THEOREM 2.2. Let $\mathrm{H} 1, \mathrm{H} 2$ hold and, in (1.2), $L_{\varepsilon}=L$ be independent of $\varepsilon$; then $\lim _{\varepsilon \rightarrow 0^{+}} V_{\varepsilon}(t, x)=V(t, x)$ uniformly on any bounded subset of $[0, T] \times X$.

PrOOF. We give a proof by contradiction. Suppose the assertion is not true. Then

there is a constant $\sigma^{\prime}>0$, a bounded subset $\mathscr{O}:[0, T] \times B_{R}(0)$, $\left(B_{R}(0)=\{x \in X \mid\|x\|<R\}\right)$ and a subsequence $\left\{\varepsilon_{k}\right\} \subset\{\varepsilon\}$, such $\}$ that $\varepsilon_{k}<1 / k$ and $\sup \left\{V_{\varepsilon_{k}}(t, x)-V(t, x):(t, x) \in \mathscr{O}\right\}>\sigma^{\prime}>0$.

Take $\delta_{0}=\min \left\{\sigma^{\prime} /(6 L+6 \bar{L}), 2 R /\left(2 A_{1} R+B_{1}\right)\right\}$. If $\left|t_{1}-t_{2}\right|+\left\|x_{1}-x_{2}\right\| \leq \delta_{0}$, then we have

$$
\begin{aligned}
\left|V_{\varepsilon}\left(t_{1}, x_{1}\right)-V_{\varepsilon}\left(t_{2}, x_{2}\right)\right| & \leq \sigma^{\prime} / 6, \\
\left|V\left(t_{1}, x_{1}\right)-V\left(t_{2}, x_{2}\right)\right| & \leq \sigma^{\prime} / 6 .
\end{aligned}
$$

Combining (2.7)-(2.9) we get

$$
\sup \left\{V_{\varepsilon_{k}}(t, x)-V(t, x):(t, x) \in\left[\delta_{0} / 2, T\right] \times B_{R}(0)\right\}>\sigma^{\prime} / 2>0 .
$$

Let $m=\left[2 T / \delta_{0}\right], S_{i}^{\prime}=\left[i \delta_{0} / 2,(i+1) \delta_{0} / 2\right] \times B_{R}(0), T_{i}^{\prime}=(i+1) T, 1 \leq i \leq m$ and $S_{m}^{\prime}=\left[T-\delta_{0} / 2, T\right) \times B_{R}(0)$; then $\left[\delta_{0} / 2, T\right) \times B_{R}(0)=\cup_{i=1}^{m} S_{i}^{\prime}$. Because $V_{\varepsilon_{k}}(T, x)-V(T, x)=0$ and $(2.10)$ holds, there exists some $1 \leq i_{0} \leq m$ such that

$$
\varlimsup_{k \rightarrow \infty} \sup \left\{V_{\varepsilon_{k}}(t, x)-V(t, x):(t, x) \in S_{i_{0}}^{\prime}\right\}>0
$$


and

$$
\varlimsup_{k \rightarrow \infty} \sup \left\{V_{\varepsilon_{k}}\left(T_{i_{0}}^{\prime}, x\right)-V\left(T_{i_{0}}^{\prime}, x\right)\right\} \leq 0 .
$$

Assume $i_{0}=m$; we can deal with other cases similarly. Let

$$
\begin{gathered}
T_{0}=\delta_{0}, L_{0}=2 R / \delta_{0}, \\
\sigma=\frac{1}{2} \varlimsup_{k \rightarrow \infty} \sup \left\{V_{\varepsilon_{k}}(t, x)-V(t, x):(t, x) \in S_{m}^{\prime}\right\}>0 .
\end{gathered}
$$

We replace $\{k\}$ by a subsequence of $\{k\}$, if necessary, such that

$$
\sup \left\{V_{\varepsilon_{k}}(t, x)-V(t, x):(t, x) \in S_{m}^{\prime}\right\}>\sigma>0, \forall k .
$$

Because

$$
\begin{aligned}
S_{m}^{\prime} & \subset\left\{(t, x) \in\left[T-\delta_{0} / 2, T\right) \times X:\|x\|<L_{0}\left(t-T+T_{0}\right)\right\} \\
& \subset S_{1}=\left\{(t, x) \in\left(T-T_{0}, T\right) \times X:\|x\|<L_{0}\left(t-T+T_{0}\right)\right\},
\end{aligned}
$$

then

$$
\sup \left\{V_{\varepsilon_{k}}(t, x)-V(t, x):(t, x) \in S_{1}\right\}>\sigma>0 .
$$

By (2.4) and (2.13), $\forall(t, x) \in S_{1} ; p, q \in X^{*}$ we have

$$
\begin{aligned}
|\bar{H}(t, x, p)-\bar{H}(t, x, q)| & \leq\left(A_{1}\|x\|+B_{1}\right)\|p-q\| \\
& \leq\left[A_{1} L_{0}\left(t-T+T_{0}\right)+B_{1}\right]\|p-q\| \\
& \leq\left(A_{1} L_{0} T_{0}+B_{1}\right)\|p-q\| \leq L_{0}\|p-q\| .
\end{aligned}
$$

Similarly,

$$
|H(t, s, x, p)-H(t, s, x, q)| \leq L_{0}\|p-q\| .
$$

We split the following proof into several steps.

Step 1. Definition of auxiliary functions and sets.

\section{Define}

$$
S=\left\{(t, x, y) \in\left(T-T_{0}, T\right) \times X \times X:\|x\|,\|y\|<L_{0}\left(t-T+T_{0}\right)\right\} .
$$

Take $\beta, \delta>0$ with $\beta+\delta<L_{0} T_{0}$. Let

$$
\begin{aligned}
d(x, y) & =\|x-y\|, \\
v(x) & =\left(\beta+\|x\|^{2}\right)^{1 / 2} .
\end{aligned}
$$


By (1.2) and (2.6) (theorem assumptions), $V_{\varepsilon}(\cdot, \cdot)$ is locally bounded. Therefore, we can choose $K>0$ such that

$$
K>\sup \left\{V_{\varepsilon_{k}}(t, x)-V(t, y):(t, x, y) \in S\right\} .
$$

Let $G(\cdot) \in C^{\infty}\left(R^{1}\right)$, which satisfies

$$
G^{\prime}(r) \geq 0, \quad G(r)= \begin{cases}0, & r \leq-\delta \\ 2 K, & r \geq 0 .\end{cases}
$$

For $0<\alpha<1, \lambda>0$, we set

$$
\begin{aligned}
\phi(t, x, y)= & V_{\varepsilon_{k}}(t, x)-V(t, y) \\
& -\left\{d(x, y)^{2} / \alpha+G\left(v(x)-L_{0}\left(t-T+T_{0}\right)\right)-\lambda(t-T)\right\} .
\end{aligned}
$$

Step 2. Properties of $\phi(t, x, y)$.

By (2.17) and (2.22), we see that $\bar{S}$ (the closure of $S$ ) is bounded, convex and $\phi(\cdot, \cdot, \cdot)$ is bounded, continuous on $\bar{S}$. According to Stegall [12],

there are elements $a_{k} \in R^{1} ; p_{k}, q_{k} \in X^{*}$ which satisfy $\left|a_{k}\right|+\left\|p_{k}\right\|+$ $\left\|q_{k}\right\|<\alpha$, such that $(t, x, y) \rightarrow \phi(t, x, y)+a_{k} t+\left\langle p_{k}, x\right\rangle+\left\langle q_{k}, y\right\rangle$ attains its maximum over $\bar{S}$ at some point $\left(t_{k}, x_{k}, y_{k}\right)$.

Let

$$
\varphi(t, x, y)=\phi(t, x, y)+a_{k} t+\left\langle p_{k}, x\right\rangle+\left\langle q_{k}, y\right\rangle
$$

and so

$$
\varphi\left(t_{k}, x_{k}, y_{k}\right) \geq \varphi\left(t_{k}, x_{k}, x_{k}\right) .
$$

Using (2.22), (2.23) and (2.24) we have

$$
d\left(x_{k}, y_{k}\right)^{2} / \alpha \leq V\left(t_{k}, x_{k}\right)-V\left(t_{k}, y_{k}\right)+4\left(L_{0}+1\right) T \alpha .
$$

By (2.6), we can get

$$
\begin{aligned}
d\left(x_{k}, y_{k}\right) & \leq L_{1} \alpha^{1 / 2}, \\
d\left(x_{k}, y_{k}\right)^{2} / \alpha & \leq L_{2} \alpha^{1 / 2},
\end{aligned}
$$

where $L_{1}, L_{2}$ are constants which are only dependent on $L_{0}, T_{0}, L$ and $\bar{L}$. Let

$$
M_{\varepsilon_{k}}=\sup \{\phi(t, x, y):(t, x, y) \in S\} .
$$


Set

$$
S_{\beta, \delta}=\left\{(t, x) \in S_{1}:\left(\beta+\|x\|^{2}\right)^{1 / 2} \leq L_{0}\left(t-T+T_{0}\right)-\delta\right\}
$$

We can choose $\beta$ and $\delta$ so small that $S_{\beta, \delta} \neq \emptyset$. Since

$$
G\left(\nu(x)-L_{0}\left(t-T+T_{0}\right)\right)=0, \quad \forall(t, x) \in S_{\beta, \delta} .
$$

Using (1.2), (2.6), (2.14) and (2.22), we choose $\beta, \delta$ and $\lambda$ so small that

$$
\begin{aligned}
& \sup \left\{\phi(t, x, x):(t, x) \in S_{\beta, \delta}\right\} \\
& \quad=\sup \left\{V_{\varepsilon_{k}}(t, x)-V(t, x)+\lambda(t-T):(t, x) \in S_{\beta, \delta}\right\}>\sigma / 2 .
\end{aligned}
$$

By (2.29), for any $k$, we have

$$
M_{\varepsilon_{k}}>\sigma / 2
$$

Consider the following three possible cases

(I) $t_{k} \rightarrow T$ or

(II) $L_{0}\left(t_{k}-T+T_{0}\right)-\max \left\{\left\|x_{k}\right\|,\left\|y_{k}\right\|\right\} \rightarrow 0$ or

(III) for some $\eta>0, N_{1}>0$, if $k \geq N_{1}$, then $\eta<t_{k}<T-\eta$; $\left\|x_{k}\right\|$, $\left\|y_{k}\right\|<$ $L_{0}\left(t_{k}-T+T_{0}\right)-\eta$.

By passing to a subsequence of $\left\{\left(t_{k}, x_{k}, y_{k}\right)\right\}$ if necessary, we can always reduce to a case in which one of (I)-(III) holds.

Step 3. We claim that we can choose $\alpha$ so small that case (I) is impossible.

In fact, noticing that $V_{\varepsilon_{k}}(T, x)=V(T, x)=g(x), \forall x \in X$, by (2.31), (2.29), (2.23), $(2.22),(1.2)$ and $(2.6)$, we have

$$
\begin{aligned}
\sigma / 2<M_{\varepsilon_{k}} \leq & \phi\left(t_{k}, x_{k}, y_{k}\right)+L_{1} \alpha \\
\leq & V_{\varepsilon_{k}}\left(t_{k}, x_{k}\right)-V\left(t_{k}, y_{k}\right)+L_{1} \alpha \\
\leq & \left(V_{\varepsilon_{k}}\left(t_{k}, x_{k}\right)-V_{\varepsilon_{k}}\left(T, x_{k}\right)\right)+\left(V_{\varepsilon_{k}}\left(T, x_{k}\right)-V\left(t_{k}, y_{k}\right)\right) \\
& -\left(V\left(T, x_{k}\right)-V\left(T, y_{k}\right)\right)+L_{1} \alpha \\
\leq & (L+\bar{L})\left|T-t_{k}\right|+L_{1} \bar{C} \alpha^{1 / 2}+L_{1} \alpha
\end{aligned}
$$

From the above, we see that case (I) is not possible if we choose $\alpha$ small enough. Thus, our claim holds.

Step 4. We claim that case (II) is not possible provided that $\alpha$ is small enough. Using (2.31), (2.29) and (2.22), we have

$$
\begin{aligned}
\sigma / 2<M_{\varepsilon_{k}} & \leq \phi\left(t_{k}, x_{k}, y_{k}\right)+L_{1} \alpha \\
& \leq V_{\varepsilon_{k}}\left(t_{k}, x_{k}\right)-V\left(t_{k}, y_{k}\right)-G\left(\nu\left(x_{k}\right)-L_{0}\left(t_{k}-T+T_{0}\right)\right)+L_{1} \alpha .
\end{aligned}
$$


If $L_{0}\left(t_{k}-T+T_{0}\right)-\left\|x_{k}\right\| \rightarrow 0$, then

$$
G\left(v\left(x_{k}\right)-L_{0}\left(t_{k}-T+T_{0}\right)\right)=-2 K .
$$

By (2.20), we have

$$
\sigma / 2 \leq-K+L_{1} \alpha .
$$

If $\alpha$ is small enough, we get a contradiction. Similarly, if $L_{0}\left(t_{k}-T+T_{0}\right)-\left\|y_{k}\right\| \rightarrow 0$, we also get a contradiction. Hence, our claim holds.

Step 5. Finally we consider case (III). Let

$$
\begin{aligned}
\Delta= & \left\{(t, x, y) \in\left(T-T_{0}, T\right) \times X \times X: \eta<t<T-\eta ;\right. \\
& \left.\|x\|,\|y\|<L_{0}\left(t-T+T_{0}\right)-\eta\right\} .
\end{aligned}
$$

Let case (III) hold, then there exists $\bar{r}>0$ which is independent of $k$, such that

$$
S_{k}=\left\{(t, x, y) \in S:\left|t-t_{k}\right|^{2}+\left\|x-x_{k}\right\|^{2}+\left\|y-y_{k}\right\|^{2} \leq \bar{r}^{2}\right\} \subset S,
$$

and $S_{k} \neq \emptyset$. Let

$$
\bar{\phi}(t, x, y)=d(x, y)^{2} / \alpha+G\left(v(x)-L_{0}\left(t-T+T_{0}\right)\right)
$$

and so

$$
\begin{aligned}
\bar{\phi}_{t}(t, x, y) & =-L_{0} G^{\prime}\left(v(x)-L_{0}\left(t-T+T_{0}\right)\right), \\
\bar{\phi}_{x}(t, x, y) & =2 d(x, y) d_{x}(x, y) / \alpha+G^{\prime}\left(v(x)-L_{0}\left(t-T+T_{0}\right)\right) D \nu(x) \\
& =2(x-y) / \alpha+G^{\prime}\left(\nu(x)-L_{0}\left(t-T+T_{0}\right)\right) x / v(x), \\
\bar{\phi}_{y}(t, x, y) & =2 d(x, y) d_{y}(x, y) / \alpha=-2(x-y) / \alpha .
\end{aligned}
$$

Let

$$
\frac{d r_{1}(s)}{d s}=\bar{H}\left(t_{k}, x_{k}, \bar{\phi}_{x}\left(t_{k}, x_{k}, y_{k}\right)\right)-H\left(t_{k}, s, x_{k}, \bar{\phi}_{x}\left(t_{k}, x_{k}, y_{k}\right)\right) \text {. }
$$

By assumptions $\mathrm{H} 1$ and $\mathrm{H} 2$, we know that $r_{1}(\cdot)$ is periodic with period 1 and $r_{1}(\cdot) \epsilon$ $C^{1}\left(R^{1}\right)$. Since $G \in C^{\infty}\left(R^{1}\right)$, there exists a constant $\bar{C}$ such that

$$
\sup \left\{\left|G^{\prime}(r)\right|, r \leq L_{0} T\right\}<\bar{C} .
$$

By (2.35), we have

$$
\begin{aligned}
& \bar{H}\left(t_{k}, x_{k}, \bar{\phi}_{x}\left(t_{k}, x_{k}, y_{k}\right)\right)-H\left(t_{k}, s, x_{k}, \bar{\phi}_{x}\left(t_{k}, x_{k}, y_{k}\right)\right) \\
& \quad \leq\left[\left\|\phi_{x}\left(t_{k}, x_{k}, y_{k}\right)\right\|+1\right] L\left(1+\left\|x_{k}\right\|\right) \\
& \quad \leq\left(2 L_{0} T_{0} / \alpha+\bar{C}+1\right)\left(1+L_{0} T_{0}\right) L .
\end{aligned}
$$


Hence $r_{1}(\cdot)$ is uniformly bounded in $k$. Assume for any $k$, sup $\left|r_{1}(\cdot)\right|<\vec{R}(\bar{R}$ may be dependent on $\alpha$ ). Consider

$$
\begin{aligned}
\psi(t, x, y)= & \varphi(t, x, y)-\varepsilon_{k} r_{1}\left(t / \varepsilon_{k}\right) \\
& -3\left[\bar{R} /\left(k \bar{r}^{2}\right)\right]\left(d\left(x, x_{k}\right)^{2}+d\left(y, y_{k}\right)^{2}+\left|t-t_{k}\right|^{2}\right)
\end{aligned}
$$

and so

$$
\psi\left(t_{k}, x_{k}, y_{k}\right) \geq \varphi\left(t_{k}, x_{k}, y_{k}\right)-\bar{R} / k \geq \varphi(t, x, y)-\bar{R} / k .
$$

As $(t, x, y) \in \partial S_{k}$, we have

$$
\begin{aligned}
\psi(t, x, y) & =\varphi(t, x, y)-\varepsilon_{k} r_{1}\left(t / \varepsilon_{k}\right)-3 \bar{R} / k \\
& \leq \varphi(t, x, y)-2 \bar{R} / k \\
& \leq \psi\left(t_{k}, x_{k}, y_{k}\right)-\bar{R} / k .
\end{aligned}
$$

According to Stegall [12], for any $\theta>0$,

there are elements $a_{2} \in R^{1}$ and $p_{2}, q_{2} \in X^{*}$ which satisfy $\left|a_{2}\right|+\mid$ $\left\|p_{2}\right\|+\left\|q_{2}\right\| \leq \min \{\theta, \alpha\}$ such that $\left.(t, x, y) \rightarrow \psi(t, x, y)+a_{2} t+\right\}$ $\left\langle p_{2}, x\right\rangle+\left\langle q_{2}, y\right\rangle$ attains its maximum over $S_{k}$ at some point $\left.(\bar{t}, \bar{x}, \bar{y}).\right)$

Take $\theta<\bar{R} /\left[k\left(4 L_{0} T_{0}+T\right)\right]$; by (2.38), the maximum point must be an interior point of $S_{k}$. Let

$$
\begin{gathered}
Z(t, x, y)=V_{\varepsilon_{k}}(t, x)-V(t, y), \\
a=a_{k}+a_{2}, p=p_{k}+p_{2}, q=q_{k}+q_{2} .
\end{gathered}
$$

Therefore $(t, x, y) \rightarrow Z(t, x, y)-\left\{\bar{\phi}(t, x, y)-\lambda(t-T)+\varepsilon_{k} r_{1}\left(t / \varepsilon_{k}\right)+3\left[\bar{R} /\left(k \bar{r}^{2}\right)\right] \times\right.$ $\left.\left(d\left(x, x_{k}\right)^{2}+d\left(y, y_{k}\right)^{2}+\left|t-t_{k}\right|^{2}\right)-a t-\langle p, x\rangle-\langle q, y\rangle\right\}$ attains its maximum over $S_{k}$ at $(\bar{t}, \bar{x}, \bar{y})$. By Lemma 2.1 and the definition of the viscosity solution, we have

$$
\begin{aligned}
\lambda \leq & \bar{\phi}_{t}(\bar{t}, \bar{x}, \bar{y})+\frac{d r_{1}\left(\bar{t} / \varepsilon_{k}\right)}{d s}+6\left[\bar{R} /\left(k \bar{r}^{2}\right)\right]\left|\bar{t}-t_{k}\right|-a \\
& +H\left(\bar{t}, \bar{t} / \varepsilon_{k}, \bar{x}, \bar{\phi}_{x}(\bar{t}, \bar{x}, \bar{y})+6\left[\bar{R} /\left(k \bar{r}^{2}\right)\right]\left(\bar{x}-x_{k}\right)-p\right) \\
& -\bar{H}\left(\bar{t}, \bar{y},-\bar{\phi}_{y}(\bar{t}, \bar{x}, \bar{y})-6\left[\bar{R} /\left(k \bar{r}^{2}\right)\right]\left(\bar{y}-y_{k}\right)+q\right) .
\end{aligned}
$$

Thus

$$
\begin{aligned}
\lambda \leq & \bar{\phi}_{t}(\bar{t}, \bar{x}, \bar{y})+6\left[\bar{R} /\left(k \bar{r}^{2}\right)\right]\left|t-t_{k}\right|-a+\left\{\bar{H}\left(t_{k}, x_{k}, \bar{\phi}_{x}\left(t_{k}, x_{k}, y_{k}\right)\right)\right. \\
& \left.-\bar{H}\left(t_{k}, y_{k},-\bar{\phi}_{y}\left(t_{k}, x_{k}, y_{k}\right)-6\left[\bar{R} /\left(k \bar{r}^{2}\right)\right]\left(\bar{y}-y_{k}\right)+q\right)\right\} \\
& +\left\{H\left(\bar{t}, \bar{t} / \varepsilon_{k}, \bar{x}, \bar{\phi}_{x}(\bar{t}, \bar{x}, \bar{y})+6\left[\bar{R} /\left(k \bar{r}^{2}\right)\right]\left(\bar{x}-x_{k}\right)-p\right)\right. \\
& \left.-H\left(t_{k}, \bar{t} / \varepsilon_{k}, x_{k}, \bar{\phi}_{x}\left(t_{k}, x_{k}, y_{k}\right)\right)\right\} .
\end{aligned}
$$


Noticing that $G \in C^{\infty}\left(R^{1}\right), \bar{\phi}_{x}, \bar{\phi}_{y}$ are Lipschitz continuous in all arguments and combining (2.34)-(2.36) with the assumptions, we have

$$
\lambda \leq A_{3} \bar{r}+A_{4} \bar{r} / \alpha+16 \bar{R} /(k \bar{r})+3 \alpha,
$$

where $A_{3}, A_{4}$ are constants which are independent of $\alpha, k$ and $\bar{r}$. In the above, first let $k \rightarrow \infty$, then let $\bar{r} \rightarrow 0$, finally let $\alpha \rightarrow 0$; then we get

$$
\lambda \leq 0 \text {. }
$$

Thus, we obtain a contradiction. So Theorem 2.2 is proved.

\section{Application: the limit problem of the value function for an optimal control problem}

Consider the following state equation in Hilbert space $X$ :

$$
\begin{cases}\frac{d x(r)}{d r}=A x(r)+f(r, r / \varepsilon, x(r), u(r)), & r \in(t, T], \\ x(t)=x, & x \in X,\end{cases}
$$

where $A: \mathscr{D}(A) \subset X \rightarrow X$ is the generator of some $C_{0}$ semigroup $e^{A t}$ and $f$ : $[0, T] \times R^{1} \times X \times U \rightarrow X$ is a given map with $U$ being a metric space in which the control $u(\cdot)$ takes values. Let

$$
\mathscr{U}[0, T]=\{u(\cdot):[0, T] \rightarrow U \mid u(\cdot) \text { measurable }\} .
$$

The cost function is given by

$$
J_{t, x}^{\varepsilon}(u(\cdot))=\int_{t}^{T} f^{0}\left(r, \frac{r}{\varepsilon}, x(r), u(r)\right) d r+g(x(T)) ;
$$

the value function is defined by

$$
V_{\varepsilon}(t, x)=\inf _{u(\cdot) \in \mathscr{U}[t, T]} J_{t . x}^{\varepsilon}(u(\cdot)) .
$$

Assume $f(t, \cdot, x, u), f^{0}(t, \cdot, x, u)$ are periodic with period 1 . Our aim is to see whether the limit of $V_{\varepsilon}$ exists as $\varepsilon \rightarrow 0^{+}$and, if the limit exists, how to determine it.

In this section, we need the following assumptions.

H3. Assume $f:[0, T] \times R^{+} \times X \times U \rightarrow X, f^{0}:[0, T] \times R^{+} \times X \times U \rightarrow$ $R^{1}\left(R^{+}=[0, \infty)\right)$ and $g: X \rightarrow R^{1}$ are continuous; $f(t, \cdot, x, u)$ and $f^{0}(t, \cdot, x, u)$ are periodic with period 1. There exists a constant $L>0$ and a continuous function 
$\omega: R^{+} \times R^{+} \rightarrow R^{+}$which is increasing in all arguments with $\omega(r, 0)=0, \forall r \geq 0$. For any $x, \bar{x} \in X ; t, \bar{t} \in[0, T] ; s, \bar{s} \in R^{+} ; u \in U$, the following hold:

$$
\begin{aligned}
& \left\{\begin{array}{l}
\|f(t, s, x, u)-f(\bar{t}, \bar{s}, \bar{x}, u)\| \leq L(|t-\bar{t}|+\|x-\bar{x}\|) \\
\\
\|f(t, s, 0, u)\| \leq L,
\end{array}\right. \\
& \left\{\begin{array}{l}
\left.\left|f^{0}(t, s, x, u)-f^{0}(\bar{t}, s, \bar{x}, u)\right| \leq L(|t-\bar{t}|+\|x\|,\|\bar{x}\|\},|s-\bar{s}|\right), \\
\left|f^{0}(t, s, 0, u)\right| \leq L,
\end{array}\right. \\
& \left\{\begin{array}{l}
|g(x)-g(\bar{x})| \leq L\|x-\bar{x}\|, \\
|g(0)| \leq L .
\end{array}\right.
\end{aligned}
$$

H4. Assume that there exists a sequence of linear bounded operators $\left\{A_{\mu}, \mu \subset\right.$ $(0,+\infty)\}, \sup \mu=+\infty$, for any $z(\cdot) \in C([0, T] ; X)$,

$$
\lim _{\mu \rightarrow+\infty} \sup _{0 \leq s \leq t \leq T}\left\|\int_{s}^{t}\left[e^{A(t-r)}-e^{A_{\mu}(t-r)}\right] f\left(r, \frac{r}{\varepsilon}, z(r), u(r)\right) d r\right\|=0
$$

uniformly in $u(\cdot) \in \mathscr{U}, \varepsilon \in(0,+\infty)$. For any $x \in X, \lim _{\mu \rightarrow+\infty}\left\|e^{A_{\mu} t} x-e^{A t} x\right\|=0$ uniformly in $t \in[0, T]$, and $\left\|e^{A_{\mu} t}\right\| \leq L e^{\omega^{\prime} t}, t \in[0, T], \mu>0$, where $\omega^{\prime}$ is a constant.

Consider the following optimal control problem:

$$
\begin{cases}\dot{x}^{\mu}(r)=A_{\mu} x^{\mu}(r)+f\left(r, r / \varepsilon, x^{\mu}(r), u(r)\right), & r \in(t, T], \\ x^{\mu}(t)=x, & x \in X .\end{cases}
$$

The cost function is given by

$$
J_{t, x}^{\mu, \varepsilon}(u(\cdot))=g\left(x^{\mu}(T)\right)+\int_{t}^{T} f^{0}\left(r, \frac{r}{\varepsilon}, x^{\mu}(r), u(r)\right) d r
$$

the value function is defined by

$$
V_{\varepsilon}^{\mu}(t, x)=\inf _{u(\cdot) \in \mathscr{U}} J_{t, x}^{\mu, \varepsilon}(u(\cdot))
$$

THEOREM 3.1. Let $\mathrm{H} 3, \mathrm{H} 4$ hold. $V_{\varepsilon}^{\mu}(\cdot, \cdot)$ is a unique viscosity solution of the $\mathrm{HJB}$ equation

$$
\begin{cases}V_{t}(t, x)+H_{\mu}\left(t, t / \varepsilon, x, V_{x}(t, x)\right)=0, & \forall(t, x) \in[0, T) \times X, \\ V(T, x)=g(x), & x \in X,\end{cases}
$$

where $H_{\mu}(t, s, x, p)=\left\langle p, A_{\mu} x\right\rangle+\inf _{u \in U}\left\{p \cdot f(t, s, x, u)+f^{0}(t, s, x, u)\right\}$.

The proof of Theorem 3.1 can be seen in $[1,7,8]$. 
THEOREM 3.2. Let $\mathrm{H} 3, \mathrm{H} 4$ hold. Then for any $R>0$ there exist constants $M$ and $C_{1}$, which are dependent on $R$, such that

(i) $\left|V_{\varepsilon}^{\mu}(t, x)\right| \leq M$,

(ii) $\left|V_{\varepsilon}^{\mu}(t, x)-V_{\varepsilon}^{\mu}(\bar{t}, \bar{x})\right| \leq C_{1}(|t-\bar{t}|+\|x-\bar{x}\|)$,

$\forall \varepsilon \in R^{+} ; t, \bar{t} \in[0, T] ;\|x\|,\|\bar{x}\| \leq R$.

The proof of the theorem is similar to the proof of [1, Lemma 1.2].

Define

$$
\begin{aligned}
& \bar{f}(t, x, u(\cdot))=\int_{0}^{1} f(t, s, x, u(s)) d s, \\
& \overline{f^{0}}(t, x \cdot u(\cdot))=\int_{0}^{1} f^{0}(t, s, x, u(s)) d s,
\end{aligned}
$$

where $u(\cdot) \in \mathscr{A}=\{u:[0,1] \rightarrow U \mid u$ measurable $\}$.

Define the averaging of the Hamiltonian

$$
\begin{array}{r}
\bar{H}_{\mu}(t, x, p)=\inf _{u(\cdot) \in \infty}\left\{p \cdot \bar{f}(t, x, u(\cdot))+\overline{f^{0}}(t, x, u(\cdot))\right\}+\left\langle p, A_{\mu} x\right\rangle, \\
\forall(t, x, p) \in[0, T] \times X \times X^{*} .
\end{array}
$$

Lemma 3.3. Let $\mathrm{H} 3, \mathrm{H} 4$ hold. Then $\forall(t, x, p) \in[0, T] \times X \times X^{*}$,

$$
\int_{0}^{1} H_{\mu}(t, s, x, p) d s=\bar{H}_{\mu}(t, x, p) .
$$

Proof. Define the functional

$$
J(y)=\int_{y}^{1}\left\{p \cdot f(t, r, x, u(r))+f^{0}(t, r, x, u(r))\right\} d r .
$$

Defined the value function

$$
U(y)=\inf _{u(\cdot) \in \infty} J(y)
$$

According to [6], we know that $U(\cdot)$ is a unique viscosity solution of the HJB equation

$$
\left\{\begin{array}{l}
\frac{d U(y)}{d y}+\inf _{u \in U}\left\{p \cdot f(t, y, x, u)+f^{0}(t, y, x, u)\right\}=0, \quad y \in[0,1) \\
U(1)=0
\end{array}\right.
$$

Obviously, $U(y)=\int_{y}^{1} \inf _{u \in U}\left\{p \cdot f(t, r, x, u)+f^{0}(t, r, x, u)\right\} d r \in C^{1}[0,1]$. Thus, the Lemma holds. 
By Lemma 3.3 and $\mathrm{H} 3, \mathrm{H} 4$ we have the following theorem.

THEOREM 3.4. Let H3, H4 hold. Then $\lim _{\varepsilon \rightarrow 0^{+}} V_{\varepsilon}^{\mu}(t, x)=\overline{V^{\mu}}(t, x), \forall(t, x) \in$ $[0, T] \times X$ and $\overline{V^{\mu}}$ is a unique viscosity solution of the $\mathrm{HJB}$ equation

$$
\left\{\begin{array}{c}
V_{t}(t, x)+\inf _{u(\cdot) \in \mathscr{S}}\left\{D_{x} V(t, x) \bar{f}(t, x, u(\cdot))+\overline{f^{0}}(t, x, u(\cdot))\right\} \\
+\left\langle D_{x} V(t, x), A_{\mu} x\right\rangle=0, \quad \forall(t, x) \in[0, T) \times X, \\
V_{t}(T, x)=g(x), \quad x \in X .
\end{array}\right.
$$

Finally we give a method to determine the limit of the original value function.

THEOREM 3.5. Let H3, H4 hold. Then $\lim _{\mu \rightarrow \infty} \lim _{\varepsilon \rightarrow 0^{+}} V_{\varepsilon}^{\mu}(t, x)=\lim _{\varepsilon \rightarrow 0^{+}} V^{\varepsilon}(t, x), \forall(t, x)$ $\in[0, T] \times X$.

Before proving the above theorem we give the following lemma.

LEMMA 3.6. Let $\mathrm{H} 3, \mathrm{H} 4$ hold and $x_{t, x}(\cdot, u(\cdot)), x_{t, x}^{\mu}(\cdot, u(\cdot))$ stand for the solution of systems (3.1) and (3.4) respectively with the initial value being $(t, x) \in[0, T] \times X$ and the control being $u(\cdot) \in \mathscr{U}$. Then

(i) $\lim _{\mu \rightarrow \infty}\left\|x_{t, x}(r, u(\cdot))-x_{t, x}^{\mu}(r, u(\cdot))\right\|=0$ uniformly in $u(\cdot) \in \mathscr{U}, \varepsilon \in R^{+}$, $r \in[0, T]$.

(ii) $\varlimsup_{\varepsilon \rightarrow 0^{+}} \sup _{u(\cdot) \in \mathscr{U}} \sup _{t \leq r \leq T}\left\|x_{t, x}^{\mu}(r, u(\cdot))-x_{t, x}(r, u(\cdot))\right\|=0$, $\lim _{\varepsilon \rightarrow 0^{+}} \sup _{u(\cdot) \in \mathscr{U}} \sup _{t \leq r \leq T}\left\|x_{t, x}^{\mu}(r, u(\cdot))-x_{t, x}(r, u(\cdot))\right\|=0$.

ProOF. By H3, we have

$$
\begin{aligned}
\left\|x_{t, x}(r)-x_{t, x}^{\mu}(r)\right\| \leq & \left\|\left(e^{A(r-t)}-e^{A_{\mu}(r-t)}\right) x\right\| \\
& +\left\|\int_{t}^{r}\left[e^{A(r-s)}-e^{A_{\mu}(r-s)}\right] f(s, s / \varepsilon, x(s), u(s)) d s\right\| \\
& +L e^{\omega T} \int_{t}^{r}\left\|x_{t, x}(s)-x_{t, x}^{\mu}(s)\right\| d s .
\end{aligned}
$$

Using Gronwall's inequality we obtain our conclusion. Part (ii) is a corollary of (i).

PROOF OF THEOREM 3.5. For any $u(\cdot) \in \mathscr{U}$, by H3, we have $\left|J_{t, x}^{\mu, \varepsilon}(u(\cdot))-J_{t, x}^{\varepsilon}(u(\cdot))\right|$ $\leq L\left\|x_{t, x}^{\mu}(T)-x_{t, x}(T)\right\|+\int_{t}^{T}\left\|x_{t, x}^{\mu}(r)-x_{t, x}(r)\right\| d r$ and so

$$
\left|J_{t, x}^{\mu, \varepsilon}(u(\cdot))-J_{t, x}^{\varepsilon}(u(\cdot))\right| \leq \bar{C} \sup _{t \leq r \leq T}\left\|x_{t, x}^{\mu}(r)-x_{t, x}(r)\right\| .
$$


In the above, taking the infimum in $u(\cdot) \in \mathscr{U}$ on both sides respectively, we have

$$
\left|V_{\varepsilon}^{\mu}(t, x)-V_{\varepsilon}(t, x)\right| \leq \bar{C} \sup _{\mu(\cdot) \in \mathscr{U}} \sup _{t \leq r \leq T}\left\|x_{t, x}^{\mu}(r)-x_{t, x}(r)\right\| .
$$

In the above, taking the superior limit and the inferior limit in $\varepsilon \rightarrow 0^{+}$on both sides respectively, we have

$$
\begin{aligned}
& \left|\lim _{\varepsilon \rightarrow 0^{+}} V_{\varepsilon}^{\mu}(t, x)-\varlimsup_{\varepsilon \rightarrow 0^{+}} V_{\varepsilon}(t, x)\right| \\
& \leq \bar{C} \varlimsup_{\varepsilon \rightarrow 0^{+}} \sup _{u(\cdot) \in \mathscr{U}} \sup _{t \leq r \leq T}\left\|x_{t, x}^{\mu}(r)-x_{t, x}(r)\right\|, \\
& \left|\lim _{\varepsilon \rightarrow 0^{+}} V_{\varepsilon}^{\mu}(t, x)-\lim _{\varepsilon \rightarrow 0^{+}} V_{\varepsilon}(t, x)\right| \\
& \leq \bar{C} \varlimsup_{\varepsilon \rightarrow 0^{+}} \sup _{u(\cdot) \in \mathscr{U}} \sup _{t \leq r \leq T}\left\|x_{t, x}^{\mu}(r)-x_{t, x}(r)\right\| .
\end{aligned}
$$

In (3.13) and (3.14), taking the superior limit and the inferior limit in $\mu \rightarrow \infty$ on both sides respectively, we get

$$
\begin{aligned}
& \left|\lim _{\mu \rightarrow \infty}\left(\lim _{\varepsilon \rightarrow 0^{+}} V_{\varepsilon}^{\mu}(t, x)\right)-\varlimsup_{\varepsilon \rightarrow 0^{+}} V_{\varepsilon}(t, x)\right| \\
& \leq \bar{C} \lim _{\mu \rightarrow \infty} \varlimsup_{\varepsilon \rightarrow 0^{+}} \sup _{\mu \in \mathscr{U}} \sup _{t \leq r \leq T}\left\|x_{t, x}^{\mu}(r)-x_{t, x}(r)\right\|, \\
& \left|\varlimsup_{\mu \rightarrow \infty}\left(\lim _{\varepsilon \rightarrow 0^{+}} V_{\varepsilon}^{\mu}(t, x)\right)-\varliminf_{\varepsilon \rightarrow 0^{+}} V_{\varepsilon}(t, x)\right| \\
& \quad \leq \bar{C} \lim _{\mu \rightarrow \infty} \varlimsup_{\varepsilon \rightarrow 0^{+}} \sup _{\mu \in \mathscr{U}} \sup _{t \leq r \leq T}\left\|x_{t, x}^{\mu}(r)-x_{t, x}(r)\right\| .
\end{aligned}
$$

By Lemma 3.6, we know that the right side of (3.15), (3.16) equals zero, so

$$
\lim _{\mu \rightarrow \infty}\left(\lim _{\varepsilon \rightarrow 0^{+}} V_{\varepsilon}^{\mu}(t, x)\right)=\varlimsup_{\varepsilon \rightarrow 0^{+}} V_{\varepsilon}(t, x) \geq \lim _{\varepsilon \rightarrow 0^{+}} V_{\varepsilon}(t, x)=\varlimsup_{\mu \rightarrow \infty}\left(\lim _{\varepsilon \rightarrow 0^{+}} V_{\varepsilon}^{\mu}(t, x)\right) .
$$

Thus $\lim _{\varepsilon \rightarrow 0^{+}} V_{\varepsilon}(t, x)=\lim _{\mu \rightarrow \infty \varepsilon \rightarrow 0^{+}} \lim _{\varepsilon} V^{\mu}(t, x)$. Hence Theorem 3.5 holds.

Combining Theorems 3.4 and 3.5, we can determine the limit of $V_{\varepsilon}(\cdot, \cdot)$ as $\varepsilon \rightarrow 0^{+}$.

\section{Acknowledgment}

The authors would like to thank Professor Xunjing Li for his help. This work was partially supported by the Chinese NFS. 


\section{References}

[1] E. N. Barron, "Averaging in Lagrange and minimax problems of optimal control", SIAM J. Control and Optim. 31 (1993) 1630-1652.

[2] A. Bensoussan, J. L. Lions and G. Papanicolaon, Asymptotic Analysis for Periodic Structures (North Holland, New York, 1987).

[3] F. Chaplais, "Averaging and deterministic optimal control", SIAM J. Control and Optim. 25 (1987) 767-780.

[4] M. G. Crandall, L. C. Evans and P. L. Lions, "Some properties of viscosity solutions of HamiltonJacobi equations", Trans. Amer. Math. Soc. 282 (1984) 487-502.

[5] M. G. Crandall and P. L. Lions, "Viscosity solutions of Hamilton-Jacobi equations", Trans. Amer. Math. Soc. 277 (1983) 1-42.

[6] M. G. Crandall and P. L. Lions, "Hamilton-Jacobi equations in infinite dimension. I Uniqueness of viscosity solutions”, J. Funct. Anal. 62 (1985) 379-396.

[7] M. G. Crandall and P. L. Lions, "Hamilton-Jacobi equations in infinite dimension. II Existence of viscosity solutions", J. Funct. Anal. 65 (1986) 368-405.

[8] M. G. Crandall and P. L. Lions, "Hamilton-Jacobi equations in infinite dimension. IV Hamilton with unbounded linear term", J. Funct. Anal. 90 (1990) 237-283.

[9] X. J. Li and J. M. Yong, Optimal Control Theory for Infinite Dimensional Systems (Birkhäuser, Boston, 1995).

[10] P. L. Lions, Generalized Solutions of Hamilton-Jacobi Equations (Pitman, London, 1982).

[11] A. Pazy, Semigroup of Linear Operators and Application to Partial Differential Equations (Springer, New York, Berlin, 1983).

[12] C. Stegall, "Optimization of functions on certain subsets of Banach space", Math. Ann. 236 (1978) 171-176.

[13] J. M. Yong, Dynamic Programming Method and HJB Equation (Shanghai Science Technology Press, Shanghai, 1992). 